\title{
Phase Interference due to Multiple Impurities and Space-Average Resistance in Quasi-One Dimensional Nanowires
}

Nobuyuki Sano and Akiko Ueda

Institute of Applied Physics, University of Tsukuba, Tsukuba 305-8573, Japan

E-mail: sano@esys.tsukuba.ac.jp

The impurity-limited resistance in quasi-one dimensional nanowires is studied by the Nonequilibrium Green function simulations and the Lippmann-Schwinger scattering theory under various spatial configurations of localized impurities. It is shown explicitly that both phase interference and phase randomization simultaneously play a crucial role in determining the impurity-limited resistance even under the fully coherent circumstances. Consequently, the ensemble average resistance of multiple impurities under the uniform configuration of impurities becomes very close to the series resistance due to single-impurity at room temperature and, thus, each impurity could be regarded as an independent scattering center.

Semiconductor nanowires have been receiving great attention in the past few decades because of their possible applications to future electronic and photonic devices. ${ }^{1-3)}$ Among others, the locality of ionized impurities plays a crucial role for optimizing the device peformance properly; the transport properties are greatly dependent of where localized impurities are located inside the nanoscale devices. ${ }^{4,5)}$

In most cases, the transport properties in nanowires have been studied with large-scale numerical simulations such as the Nonequilibrium Green function (NEGF). However, because of the computational capability, the incorporation of the whole device structure including the dielectric surroundings is very difficult and the number of impurities included in such calculations is rather limited. In many cases, just one single-impurity is put at various locations in the cross-sectional area of the quasi-one dimensional (quasi-1D) nanowire and the impurity-limited resistance or equivalently the mobility is extracted. ${ }^{6-9)}$ There are indeed several theoretical reports in which multiple impurities are introduced explicitly to evaluate the transport properties in the tight-binding NEGF and it has been demonstrated how the device characteristics could actually fluctuate, depending on the spatial configuration of impurities. ${ }^{10-13)}$ However, it is very difficult to extract the distinct effects of phase interference due to localized impurities among others from only such bruteforce simulations.

In the present Letter, we briefly report the first systematic investigations of the impurity-limited resistance in quasi-1D nanowires by employing both the NEGF simulations and the Lippmann-Schwinger (LS) scattering theory from the viewpoint of phase interference among multiple impurities.

Following the Landauer approach, the conductance $G$ through the doped channel region of the nanowire is calculated from the transmission coefficient of the in-coming electrons from the reservoirs (source and drain). The transmission coefficient is calculated from the FisherLee formula with the retarded Green function from the NEGFs. ${ }^{14)}$ In the theoretical analyses, the asymptotic forms of the LS equation are employed to extract the transmission and reflection coefficients. ${ }^{15)}$ It should be stressed that the total resistance given by the inverse of
$G$ consists of two contributions; the contact and the channel resistances. The former is caused by the difference in the number of modes between the reservoir and the lead, whereas the latter is associated with the scattering potential by ionized impurities, phonons, surface roughness, and the long-range potential modulation. This potential modulation is, further, attributed to the long-range part of the Coulomb potential of ionized impurities/carriers as well as the applied gate voltage. In order to extract the impurity-limited resistance, we consider only impurity scattering and the resistance induced by the long-range potential modulation is eliminated by assuming that the channel potential is flat along the wire axis. As a consequence, the impurity-limited resistance $R_{s}$ is obtained by subtracting the contact resistance from the total resistance, $R_{s}=R_{t o t}-R_{0}$, and, thus, given by

$$
R_{s}=\frac{\pi \hbar}{e^{2}} \frac{1}{g_{s u b}} \frac{\int_{-\infty}^{\infty} d E \sum_{A} R_{A}(E)\left(-\frac{\partial f_{F D}(E)}{\partial E}\right)}{\int_{-\infty}^{\infty} d E \sum_{A} T_{A}(E)\left(-\frac{\partial f_{F D}(E)}{\partial E}\right)},
$$

where $g_{s u b}$ is the number of subband available for the in-coming electrons in the nanowire and $f_{F D}(E)$ is the Fermi-Dirac distribution of the reservoirs. $R_{A}(E)$ and $T_{A}(E)\left(=1-R_{A}(E)\right)$ are, respectively, the reflection and transmission coefficients for the electrons with energy $E$ through the subband $A$.

In the NEGF simulations, we employ a rectangular nanowire with the side length of $a_{c}=3.5 \mathrm{~nm}$, as schematically drawn in top of Fig. 1. Ionized donor or acceptor impurities are distributed at random in Si channel. The average impurity density in the channel is fixed at $10^{19}$ $\mathrm{cm}^{-3}$ so that the channel length $L$ varies according to the number of impurities doped in the channel, namely, $L=8 \mathrm{~nm}$ for a single impurity, $16 \mathrm{~nm}$ for two impurities, etc. Since many simulations under various impurity configurations are required to perform, the impurity scattering potential is assumed to be a simple screened Coulomb potential with the screening length of $\lambda_{s c}=1.3$ nm along with the image charges associated with the $\mathrm{Si} /$ Gate-Oxide interface.

We first show the impurity-limited resistances for two correlated donor or acceptor impurities located inside the nanowire in Fig. 1. The spatial configuration of two im- 

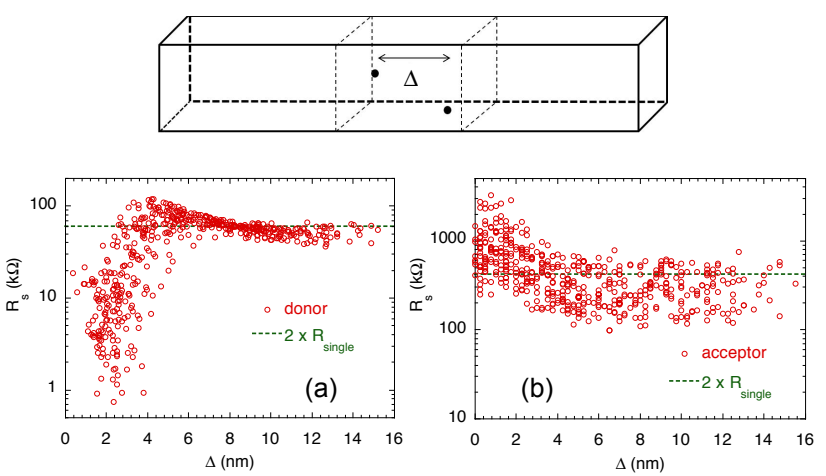

Fig. 1. (Top) Rectangular nanowire employed in this study. Two localized impurities are located at random inside the channel region. (Bottom) Impurity-limited resistance $R_{s}$ of two correlated (a) donors and (b) acceptors as a function of the impurity separation $\Delta$ along the wire axis direction. The resistances are calculated from the tight-binding NEGF and plotted for 500 different configurations of two impurities. The horizontal (green) dashed line shows the value of the series resistance of two impurities, $2 R_{\text {single }}$.

purities are generated at random and the resistances for 500 different impurity configurations are shown. There are some distinct features in the two results: The magnitude of $R_{s}$ is very different for the cases of donors and acceptors, as already noted. ${ }^{8,9)}$ This indicates the fact that the scattering is so strong under nanostructures and the Born approximation, in which impurity scattering is independent of the type of ionized impurities, breaks down. Also, $R_{s}$ in the case of donors is greatly reduced as the impurity separation $\Delta$ becomes very close to zero, compared with that of acceptors. This is attributed to the different behavior of the long-range part of the screened Coulomb potential. Namely, the scattering potential of acceptors behaves as potential barriers, whereas that of donors behaves as potential depressions. When $\Delta$ is very small, the potential depressions due to two closely located donors entirely lower the potential and the total resistance is greatly reduced.

Nevertheless, there are some common features in these two cases: There is a peak in $R_{s}$ at small $\Delta$ and then $R_{s}$ approaches some constant value at large $\Delta$. Surprisingly, this constant value is found to be quite close to the resistance of two impurities, $2 R_{\text {single }}$, with $R_{\text {single }}$ being the space-average resistance of single-impurity. In other words, the correlated $R_{s}$ of two impurities approaches the series resistance at large $\Delta$ and, thus, an uncorrelated limit, although no phase breaking scattering is involved in the present NEGF simulations.

In order to clarify the physical origin of these common features, the impurity-limited resistance is theoretically calculated from the LS equation under the same nanowire structure with the same dimensions. The only difference is that we employ a simplified short-range scattering potential $V(\mathbf{R})$ defined by

$$
V(\mathbf{R})=\sum_{r=1}^{2}\left(v_{c} a S\right) \delta^{(3)}\left(\mathbf{R}-\mathbf{R}_{0 r}\right),
$$

where $v_{c}$ is the scattering potential energy, $a$ is the characteristic length along the axis direction over which

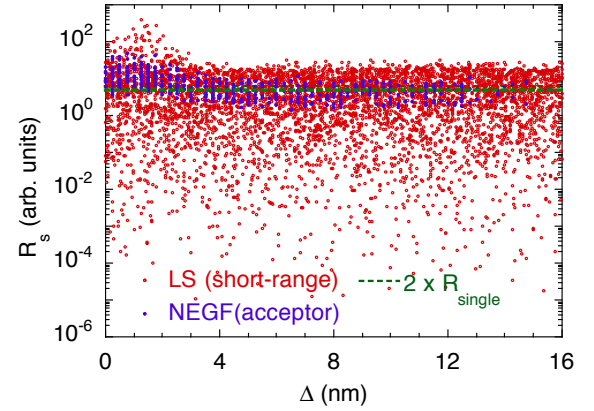

Fig. 2. Impurity-limited resistances $R_{s}$ of two impurities obtained from the LS equation. The resistances for 5000 different impurity configurations are shown. The resistances of acceptor impurities from the NEGF are also shown with solid symbols. The horizontal (green) dashed line shows the value of $2 R_{\text {single }}$ for the cases of both the LS and NEGF.

the scattering potential is effective, and $S$ is the crosssectional area of the wire. The position of the $r$-th impurity is denoted by $\mathbf{R}_{0 r}=\left(\mathbf{r}_{0 r}, z_{0 r}\right)$. For simplicity, we assume that each impurity has the same scattering potential energy of $v_{c}=282 \mathrm{meV}$, corresponding to the screening length of $\lambda_{s c}=1.3 \mathrm{~nm}$. The reflection coefficient $R_{A}(E)$ for two correlated impurities is analytically obtained under the extreme quantum limit, where only the lowest subband is involved in the electron transport, and expressed by

$$
R_{A}(E)=\frac{\Gamma_{A}(E)}{1+\Gamma_{A}(E)}
$$

with

$$
\begin{aligned}
& \Gamma_{A}(E)=\gamma_{1}^{2}+{\gamma_{2}}^{2}+2 \gamma_{1}{ }^{2} \gamma_{2}{ }^{2} \\
& \quad+2 \gamma_{1} \gamma_{2}\left\{\left(1-\gamma_{1} \gamma_{2}\right) \cos (2 k \Delta)+\left(\gamma_{1}+\gamma_{2}\right) \sin (2 k \Delta)\right\}
\end{aligned}
$$

where $k$ is the electron wavevector in the axis direction and $\Delta=\left|z_{01}-z_{02}\right|$. The scattering parameter $\gamma_{r}$ for the $r$-th impurity $(r=1,2)$ is given by

$$
\gamma_{r}\left(E, \mathbf{r}_{0 r}\right)=v_{c} \frac{a}{\hbar} \sqrt{\frac{m}{2\left(E-\varepsilon_{A}\right)}} S\left|\xi_{A}\left(\mathbf{r}_{0 r}\right)\right|^{2},
$$

where $\varepsilon_{A}$ is the lowest subband energy and $\xi_{A}\left(\mathbf{r}_{0 r}\right)$ is the subband wavefunction. The impurity-limited resistance $R_{s}$ is then calculated from Eq. (1). Figure 2 shows the resistances $R_{s}$ thus calculated from the LS theory for 5000 different configurations of two impurities. For comparison, the resistances of acceptor impurities obtained from the NEGF simulations are also shown. The magnitude of $R_{s}$ from the NEGF is adjusted such that the value of $2 R_{\text {single }}$ from the NEGF coincides with that from the LS theory. The similarity of the $\Delta$-dependence in $R_{s}$ between the two results is clear, though the results from the LS theory scatter over much greater ranges due to the short-range nature of the scattering potential.

The fluctuations of $R_{s}$ at fixed $\Delta$ result from the variations in magnitude of the subband wavefunctions and, thus, have nothing to do with the phase interference. In order to eliminate such fluctuations, we calculate $R_{s}$ of two impurities located on the wire axis from the LS 


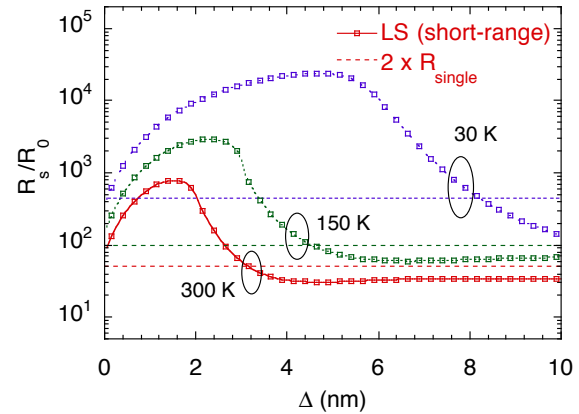

Fig. 3. Impurity-limited resistance $R_{s}$ of two impurities located on the wire axis from the LS theory as a function of the impurity separation $\Delta$ along the wire axis. $R_{s}$ is evaluated for $T=300,150$, and $30 \mathrm{~K}$ and $R_{0}$ is the quantum resistance. The horizontal dashed lines show the uncorrelated limit, $2 R_{\text {single }}$.

theory and the results are shown in Fig. 3 for the three different temperatures. $R_{0}$ is the quantum resistance and given by $R_{0}=\pi \hbar / e^{2}$. The oscillatory behavior is clearly seen at small $\Delta$. This results from the trigonometric dependence in the reflection coefficient and, thus, represents the constructive phase interference between the two impurities. This oscillation, however, damps at large $\Delta$ and $R_{s}$ approaches to a constant value at high temperature $(T=300$ and $150 \mathrm{~K})$. This asymptotic value at large $\Delta$ is indeed close to $2 R_{\text {single }}$ where $R_{\text {single }}$ is the resistance of the single-impurity placed on the wire axis and, thus, approaches the uncorrelated limit. We would like to stress that neither spatial averaging with respect to impurity configurations nor energy dissipating scattering such as phonon interaction is involved. Therefore, the phase randomization, which leads to the uncorrelated limit, takes place even under the fully coherent circumstances. From Fig. 3, this phase randomization is clearly related with temperature of the reservoirs and equivalent to the so-called "self-averaging" in impurity scattering. ${ }^{16)}$ This part will be discussed elsewhere.

Thanks to the phase randomization, the ensemble average resistance is expected to be close to the uncorrelated value of $2 R_{\text {single }}$. This is indeed true and even holds true over the great ranges of the coupling strength of impurity scattering. Figure 4 shows the ensemble average impurity-limited resistance of two impurities from the LS equation as a function of the scattering potential energy $v_{c}$. The resistance is averaged over various impurity configurations under the uniform distribution. The exact $R_{s}$ is well approximated with the uncorrelated value of $2 R_{\text {single. }}$ It does not imply that the coupling is weak so that higher-order corrections could be ignored. But rather, the coupling is so strong that the Born approximation entirely breaks down except at very small $v_{c}$, as clearly seen in Fig. 4. Therefore, as far as the spaceaverage resistance is concerned, each impurity could be regarded as uncorrelated. This is also confirmed by the more elaborate NEGF simulations. Figure 5 shows the impurity-limited resistance for 300 patterns of impurity configurations from the NEGF simulations as a function of the number of donors or acceptors. $R_{s}$ greatly varies depending on the position of impurities, but the

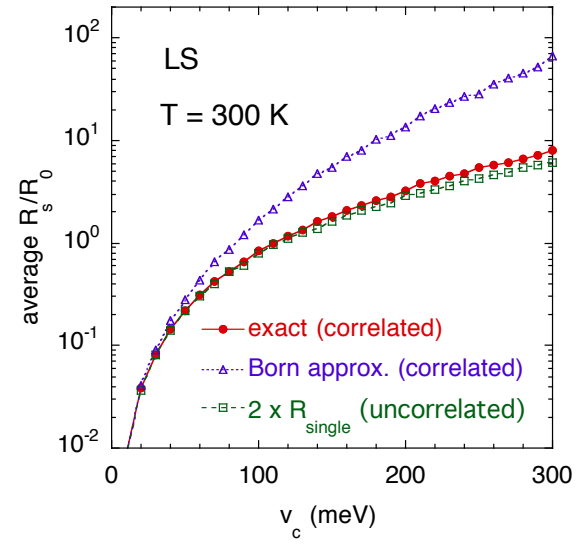

Fig. 4. Ensemble average resistance of two impurities under uniform distribution as a function of the scattering potential energy $v_{c}, R_{s}$ is calculated with the exact formula and the Born approximation from the LS equation. $2 R_{\text {single }}$ represents the uncorrelated limit, where $R_{\text {single }}$ is the ensemble average resistance of single-impurity.

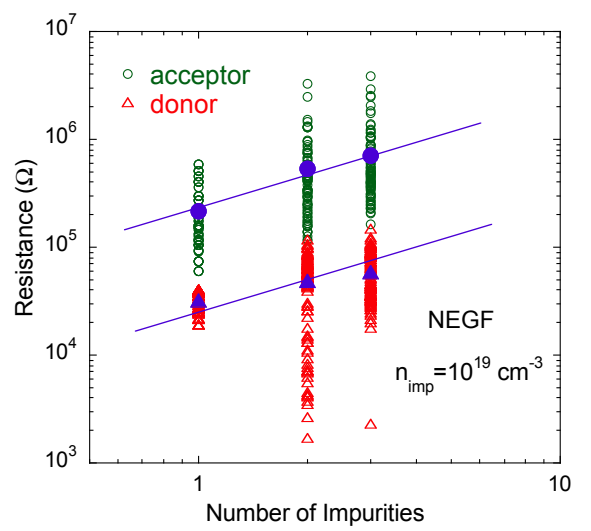

Fig. 5. Impurity-limited resistance $R_{s}$ from the NEGF simulations as a function of the number of donors or acceptors. $R_{s}$ for 300 different impurity configurations are shown. The large solid symbols represent the ensemble average of the resistances.

average value of $R_{s}$ follows the linear relationship with the number of impurities (or equivalently the channel length). Therefore, the space-average $R_{s}$ obeys the classical Ohm's law and each impurity could be regarded as an independent scattering center.

We have investigated the spatial correlation effects of ionized impurities under the quasi-1D nanowire. We have explicitly shown how the phase interference and phase randomization take place simultaneously in the impuritylimited resistance $R_{s}$ at room temperature. Also, we have found that the space-average resistance of multiple impurities under the uniform distribution becomes very close to the series resistance of single-impurity and, thus, follows the classical Ohm's law.

Acknowledgement The authors wish to thank S. Honda for his contribution in the early stage of this study. This study was supported in part by the Ministry of Education, Science, Sports, and Culture under Grant-in-Aid for Scientific Research (B) (no. 15H03983). 
1) J. Appenzeller, J. Knoch, M. Bjork, H. Riel, H. Schmid, and W. Riess, IEEE Trans. Electron Devices 55, 2827 (2008).

2) V. Schmidt, J. V. Wittemann, S. Senz, and U. Gösele, Adv. Mater. 21, 2681 (2009).

3) R. Rurali, Rev. Mod. Phys. 82, 427 (2010).

4) N. Sano, K. Matsuzawa, M. Mukai, and N. Nakayama, in Technical Digest of Int. Electron Devices Meeting, 2000, p. 275.

5) N. Sano, K. Matsuzawa, M. Mukai, and N. Nakayama, Microelectron. Reliab. 42, 189 (2002).

6) T. Markussen, R. Rurali, A.-P. Jauho, and M. Brandbyge, Phys. Rev. Lett. 99, 076803 (2007).

7) R. Rurali, T. Markussen, J. Sune, M. Brandbyge, and A.-P. Jauho, Nano Lett. 8, 2825 (2008).

8) M. P. Persson, H. Mera, Y.-M. Niquet, C. Delerue, and M. Diarra, Phys. Rev. B 82, 115318 (2010).
9) Y.-M. Niquet, H. Mera, and C. Delerue, Appl. Phys. Lett. 100, 153119 (2012).

10) A. Martinez, M. Aldegunde, N. Seoane, A. Brown, J. Barker, and A. Asenov, IEEE Trans. Electron Devices 58, 2209 (2011).

11) M. Aldegunde, A. Martinez, and J. Barker, IEEE Trans. Electron Devices 33, 194 (2012).

12) S. Sylvia, K. Habib, M. Khayer, K. Alam, M. Neupane, and R. Lake, IEEE Trans. Electron Devices 61, 2208 (2014).

13) A. Ueda, M. Luisier, and N. Sano, to be published in Appl. Phys. Lett.

14) D. S. Fisher and P. A. Lee, Phys. Rev. B 23, 6851 (1981).

15) M. DiVentra, Electrical Transport in Nanoscale Systems (Cambridge University Press, UK, 2008).

16) W. Kohn and J. M. Luttinger, Phys. Rev. 108, 590 (1957). 